\title{
Case report: nocardia infection associated with ectopic cushings
}

\author{
Azra Rizwan ${ }^{*}$, Aqiba Sarfaraz ${ }^{\dagger}$, Abdul Jabbar ${ }^{\dagger}$, Jaweed Akhter $^{\dagger}$ and Najmul Islam ${ }^{\dagger}$
}

\begin{abstract}
Background: Cushing's syndrome results from exposure to excess glucocorticoids. Ectopic Cushings is endogenous ACTH dependant form of Cushing's associated with markedly raised ACTH and cortisol levels. This leads to an impaired immune response, setting the stage for occurrence of opportunistic infections. Nocardiosis is a gram positive bacterial infection caused by aerobic actinomycetes in genus Nocardia. We report a series of patients diagnosed with ectopic Cushings, having pneumonia with Nocardia spp. In one of these cases, the manifestations of Cushing's disappeared with treatment for Nocardia.
\end{abstract}

Case presentation: Two middle aged men of Asian descent presented to the Endocrine clinic: the first with history of exertional shortness of breath, and weight loss for 1 year, the other with facial swelling, disturbed sleep and lethargy for a month. The third case was a young Asian male who presented with progressive weakness \& weight loss for 2 months. All three patients had uncontrolled hypertension, high blood sugars \& were hypokalemic (K: 2.52, 2.9, $1.5 \mathrm{mmol} / \mathrm{l}) ; 24$ hour urine cortisol was elevated at 2000, 27216 and 9088 (32-243 ug/24 hours); ACTH 68.5, 159, $255[0-48 \mathrm{pg} / \mathrm{ml})$, respectively. Their MRI pituitary was normal, inferior petrosal sinus sampling revealed no central peripheral gradient. CT chest of these subjects demonstrated cavitatory lung lesions; microscopic analysis of respiratory samples was suggestive of infection with Nocardia spp. Histopathology of bronchoscopic-guided biopsy revealed no malignancy. Antihypertensives, insulin, potassium replacement, ketoconazole \& trimethoprim-sulphamethoxazole (TS) were initiated. The patients' symptomatology improved \& cavitatory lesions resolved with treatment. The primary source for the ectopic cushings remained unknown. The first case required bilateral adrenalectomy. The second case followed a progressively downhill course leading to death. In the third case, we were able to completely taper off ketoconazole, potassium, insulin \& antihypertensives, after starting TS.

Conclusion: Opportunistic infections are known to be associated with Cushing's syndrome, and higher levels of glucocorticoid secretion are found in patients with ectopically produced ACTH. Pulmonary nocardiosis is important differential to consider. This series includes the first case reported in which signs and symptoms of cushings subsided after treatment of Nocardia.

Keywords: ACTH Adrenocorticotrophin Hormone, TS trimethoprim-sulphamethoxazole, IPSS inferior petrosal sinus sampling

\section{Background}

Cushing's syndrome results from prolonged exposure to excess glucocorticoids. This could be due to an increased endogenous production of steroids, or could result from overzealous use of medication containing steroids [1]. Ectopic Cushings is an endogenous ACTH dependant form of Cushing's that accounts for $10 \%$ of

\footnotetext{
* Correspondence: azra.rizwan@aku.edu

${ }^{\dagger}$ Equal contributors

Section of Endocrinology, Department of Medicine, Aga Khan University Hospital, Stadium Road, P.O. Box 3500, Karachi 74800, Pakistan
}

ACTH dependant causes [2]. This form of Cushing's is associated with markedly raised $\mathrm{ACTH}$, and, subsequently, cortisol levels. Elevated cortisol levels lead to an impaired immune response by neutrophils \& macrophages and diminished recruitment of these inflammatory cells into the infected site. This sets the stage for the occurrence of bacterial and fungal opportunistic infections [2]. Nocardiosis is a gram positive bacterial infection caused by aerobic actinomycetes in the genus Nocardia [3].

We report a series of patients diagnosed with ectopic Cushings, with microscopic analysis suggestive of

\section{Biomed Central}


pulmonary nocardiosis. In one of these cases, the manifestation of Cushing's disappeared simply with treatment for Nocardia.

\section{Case presentation}

Case 1

A 53 year gentleman, banker by profession, first presented to us in 2006, with exertional shortness of breath and lethargy for a period of 1 year. Associated with this was significant weight loss (6 $\mathrm{kg}$ over a 4 month period). His appetite had significantly decreased, although there had been no cough or fever. He had also been facing difficulty in climbing stairs since the previous month. Hypertension was diagnosed 6 months prior to presentation. There was no history of Tuberculosis or Type 2 diabetes. Addictions included a history of smoking 30-35 cigarettes/day for the past 25-30 years. There was no history of steroid intake, including that of traditional homeopathic or herbal medication consumed extensively in the Indo-Pak region.

On examination, his blood pressure was 160/90 $\mathrm{mmHg}$. He had a pulse of 86 beats/minute, which was regular. His weight was $71 \mathrm{~kg}$, height of $170 \mathrm{~cm}$. He was mildly anaemic, had no goiter or clinically palpable nodule. There was no gynecomastia, no striae, no evidence of easy bruisability or pruritis. His visual fields were normal; there was no papilledema. There was significant proximal myopathy. Chest examination revealed crackles at left lung base. The rest of the systemic examination was normal.

He was severely hypokalemic (K: 2.52). Screening for Cushings revealed a 24hour urine cortisol of $2000 \mathrm{ug} /$ $24 \mathrm{hrs}$ (32-243), an overnight dexamethasone suppression test of $31.7 \mathrm{ug} / \mathrm{dl}(<1.8 \mathrm{ug} / \mathrm{dl})$, while baseline ACTH was

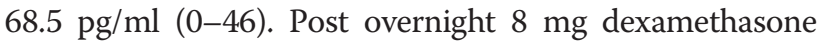
suppression test revealed a value of $18.9 \mathrm{ug} / \mathrm{dl}$ (baseline cortisol: $20 \mathrm{ug} / \mathrm{dl}$ ).

Chest radiography demonstrated left lower haziness, while CT chest with contrast revealed a cavitatory lesion
$(3.6 \times 2.5 \times 1.8 \mathrm{~cm})$ in left lower lobe lung with adjacent alveolitis (Figure 1). There was no mediastinal and hilar lymphadenopathy.

The history \& laboratory work-up appeared to be consistent with ectopic Cushings, with the obvious source being the lungs. Hence, we proceeded with a CT guided biopsy of the lungs, which was repeated a week later. On both occasions, biopsy revealed chronic inflammatory changes, with no evidence of malignancy. Given an inconclusive biopsy result, and ACTH levels that were not too high, we requested for an MRI of the pituitary to look for a pituitary adenoma (Cushing's disease). This revealed a $0.5 \times 0.5 \mathrm{~cm}$ lesion, not showing typical post contrast signal of pituitary adenoma. A subsequent IPSS (inferior petrosal sinus sampling) and sampling from other sites revealed no gradient of ACTH level (Table 1). In the meantime, trimethoprim-sulphamethoxazole (TS), at a dose of $10 \mathrm{mg}$ trimethoprim/ $\mathrm{kg} /$ day $(710 \mathrm{mg} /$ day $)$, had empirically been started for infection with Nocardia spp. by the pulmonologist keeping in mind a repeat CT Chest (Figure 1b) showing the patient's lung lesion to have almost doubled within a span of 14 days. This is a sign more consistent with infection, since the average doubling time of lung cancers reported in most studies is at least 20 days [4]. At this stage, the pulmonologist felt that a bronchoscopy would not help in the case of such a deep seated peripheral lesion. A Cardiothoracic opinion deemed the patient's overall condition too sick for a video assisted transthoracic surgery/open lung biopsy. His condition started improving slightly with conservative therapy which included potassium replacement, insulin, anti hypertensives, ketoconazole and TS, after which he was discharged on request. Two months later, he was readmitted with worsening shortness of breath, worsening proximal myopathy and bone pains. An X ray chest revealed left lower lobe collapse. A bronchoscopy was done. Gram stain and partial acid fast staining of bronchial washings was suggestive of infection with Nocardia spp.
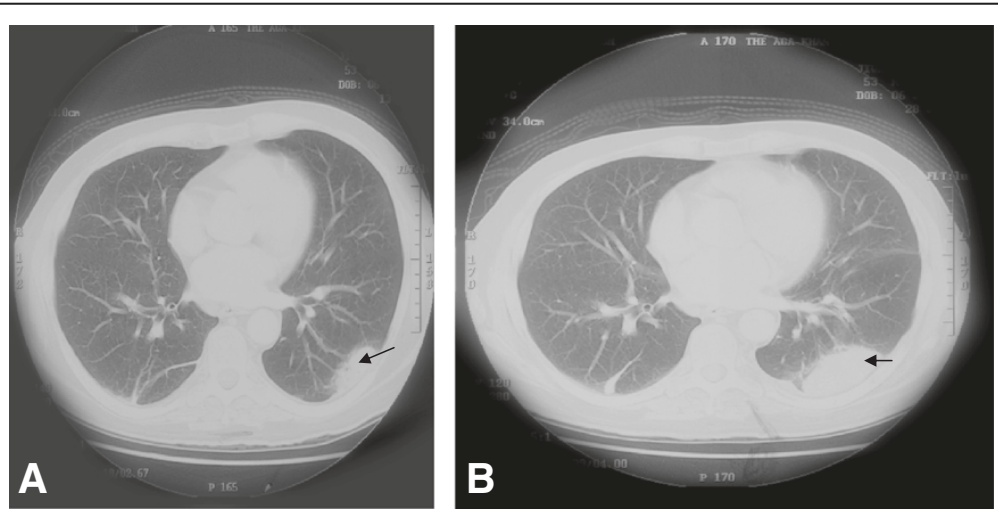

Figure 1 CT Chest before treatment for Nocardia (Case 1). (A) Cavitatory lesion $(3.6 \times 2.5 \times 1.8 \mathrm{~cm})$ in left lower lobe lung with adjacent alveolitis. (B) Soft tissue density mass left lower lobe $(5.2 \times 3.5 \times 2.8 \mathrm{~cm})$-near doubling of lesion within span of 14 days. 
Table 1 Inferior petrosal sinus sampling (Case 1)

\begin{tabular}{lll}
\hline ACTH levels & $\mathbf{1 6 / 0 7 / 2 0 0 6}$ & $\mathbf{1 3 / 1 2 / 2 0 0 6}$ \\
\hline RT INF Petrosal 1 & 127 & 67.7 \\
RT INF Petrosal 2 & 113 & 70.7 \\
LT INF Petrosal 1 & 126 & 63.5 \\
LT INF Petrosal 2 & 136 & 71.8 \\
Peripheral 1 & & 59.8 \\
Peripheral 2 & & 43.7 \\
Peripheral 3 & 113 & 81.4 \\
RT Atrium & 134 & \\
IVC & 111 & 62.5 \\
SVC & 141 & 70.5 \\
INFRA Renal & & 62.3 \\
RT Internal Jugular & & 95.2 \\
LT Internal Jugular & & 60.1
\end{tabular}

(Table 2). However, the culture did not grow Nocardia spp. possibly due to prior antibiotic treatment. The dose of TS was maximized to $15 \mathrm{mg} / \mathrm{kg} /$ day (1200 mg/day), and the medication was continued for a period of 6 months. Culture for Mycobacterium tuberculosis remained negative. The histopathology sample of the bronchoscopic guided biopsy was not consistent with malignancy. A repeat petrosal sinus sampling was done, again revealing no central-peripheral gradient (Table 1). Since the source of the ectopic cushings could not be identified and his general condition was worsening, he underwent bilateral adrenalectomy. Following the procedure, his condition slowly improved. A repeat CT chest showed resolution of the cavitatory lesion detected earlier (Figure 2).

\section{Case 2}

A 54 years old businessman presented to the Endocrine clinic. He was hypertensive, non-smoker, but tobacco pan chewer. For 3 weeks prior to presentation he had been having swelling of the feet and face, disturbance in sleep pattern, and was irritable. He reported weight loss of $10 \mathrm{~kg}$ over the previous 45 days. Medication he had been prescribed were losartan, atenolol, spironolactone.

On examination, he was found to have blood pressure of 150/90 mmHg. He was having significant pedal edema, ecchymoses, lingual ulcers and central obesity. His right leg was disproportionately swollen, and red. Laboratory investigations revealed severe hypokalemia [K:2.9] and an elevated fasting blood sugar (FBS) of $200 \mathrm{mg} / \mathrm{dl}$. An ultrasound dopplers revealed no deep venous thrombosis. He was admitted for work-up \& further management for uncontrolled hyperglycemia, hypertension, hypokalemia and right leg extremity cellulitis. His antihypertensive medication [losartan, aldactone]
Table 2 Microbiology data details

\begin{tabular}{|c|c|c|c|}
\hline & Smear & Culture & $\begin{array}{l}\text { Sensitivity } \\
\text { [S-Sensitive, } \\
\text { R-Resistant] }\end{array}$ \\
\hline Case 1 & $\begin{array}{l}\text { Suggestive of } \\
\text { Nocardia }\end{array}$ & $\begin{array}{l}\text { "Nocardia fails to } \\
\text { grow on culture" }\end{array}$ & Not applicable \\
\hline \multirow[t]{11}{*}{ Case 2} & $\begin{array}{l}\text { Suggestive of } \\
\text { Nocardia }\end{array}$ & $\begin{array}{l}\text { Growth of } \\
\text { Nocardia spp }\end{array}$ & $\begin{array}{l}\text { Amoxicillin/ } \\
\text { clavulanate - S }\end{array}$ \\
\hline & & & Ampicillin - $R$ \\
\hline & & & Amikacin-S \\
\hline & & & Imipinem-S \\
\hline & & & Gentamicin-R \\
\hline & & & Ceftriaxone-S \\
\hline & & & Cefotaxime-R \\
\hline & & & Erythromycin-R \\
\hline & & & Ofloxacin $-R$ \\
\hline & & & Co-trimoxazole-S \\
\hline & & & Tetracycline-R \\
\hline \multirow[t]{9}{*}{ Case 3} & $\begin{array}{l}\text { Suggestive of } \\
\text { Nocardia }\end{array}$ & $\begin{array}{l}\text { Growth of } \\
\text { Nocardia spp }\end{array}$ & $\begin{array}{l}\text { Amoxicillin/ } \\
\text { Clavulanate-S }\end{array}$ \\
\hline & & & Ampicillin-S \\
\hline & & & Amikacin-S \\
\hline & & & Imipinem-S \\
\hline & & & Gentamicin-S \\
\hline & & & Ceftriaxone-S \\
\hline & & & Erythromycin- S \\
\hline & & & Co-trimoxazole-S \\
\hline & & & Tetracycline-S \\
\hline
\end{tabular}

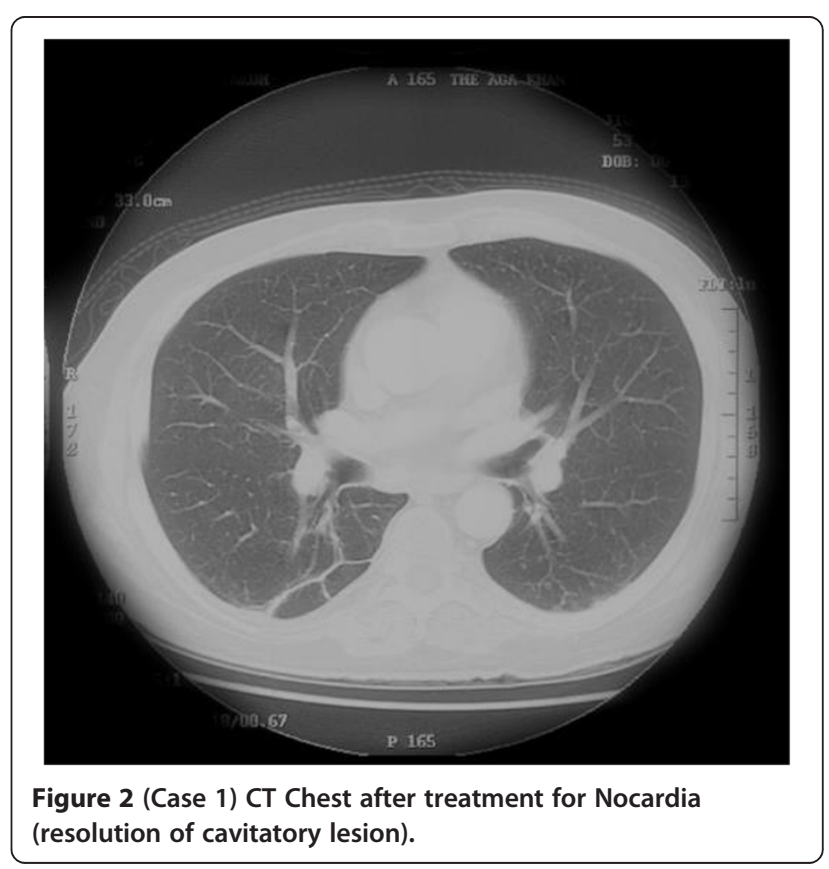


and potassium supplementation were upgraded. Subcutaneous insulin was initiated for blood sugar control.

Further work-up revealed an elevated urine cortisol (27216) and overnight dexamethasone suppression test (134 ug/dl). A subsequent ACTH level was raised at 159 (0-48). MRI pituitary was normal. His $\mathrm{x}$ ray chest revealed a cavitatory lesion adjacent to left heart border, and a possible left pleural effusion. A subsequent CT chest with contrast confirmed a left lung cavitatory lesion. A CT guided biopsy of lung lesion was done, with the section revealing blood admixed with acute inflammatory cells, predominantly neutrophils. No tumour tissue was identified. The patient then had an episode of haemoptysis, prompting bronchoscopy. No endobronchial lesion was seen. Bronchial washings revealed no malignant cells, budding yeast cells or hyphae. Moderate branching gram positive filamentous rods were seen and the modified acid fast stain was positive, which was highly suggestive of Nocardia. Subsequent culture grew Nocardia spp. (Table 2) Acid fast bacilli were not seen. A repeat $\mathrm{CT}$ scan a week later revealed development of new cavitatory lesions in the left lung upper and lower lobe.

He was started on trimethoprim-sulphamethoxazole (TS) at $15 \mathrm{mg}$ trimethoprim/ $\mathrm{kg}$ (1200 mg/day) for the nocardia, and discharged in a stable condition, with advice to continue TS for 6 months. A follow-up CT chest 3 months later revealed complete resolution of cavitatory lesions previously seen in upper \& lower lobe left lung. The patient then got lost to follow-up.

A year later, he presented with worsening bone pains, for which a whole body skeletal scintigraphy was requested. The latter showed widespread bony metastasis. $\mathrm{CT}$ chest and abdomen with contrast revealed multiple tiny nodules in both lungs and enlarged lymph nodes within the mediastinum. Multiple well-defined low attenuated lesions were noted in the liver, while 3 peripherally placed lesions identified in spleen. Both adrenals were bulky. Numerous enlarged paraaortic and peripancreatic lymph nodes were identified. A CT guided biopsy of a paraaortic lymph node detected this time showed a metastatic adenocarcinoma.
Subsequently, he was readmitted with worsening shortness of breath. Chest radiography showed hilar vascular congestion with pulmonary vascular congestion in lower lung zones bilaterally. He was started on broad spectrum intravenous antibiotics, and was given intravenous fluids judiciously. On the second day of admission, he became unresponsive, pulseless and hypotensive. Since the code had been decided as no shock and no CPR, he was not intubated, and death was declared shortly.

\section{Case 3}

A 38 year male, hypertensive for 5 years, and an exsmoker was admitted with chief complaints of progressive weakness, particularly on climbing stairs and standing from squatting position, and with excessive weight loss for 2 months. He had also been diagnosed with type 2 diabetes a month prior to presentation. On physical examination, he was afebrile with a blood pressure of 160/100 $\mathrm{mmHg}$ and respiratory rate of 22 breaths per minute. He had ecchymoses on his arms and legs. Respiratory examination revealed diminished breath sounds at the lung bases with some crackles. On neurologic examination, he was well oriented to time, place and person. Power was $3 / 5$ on both lower limbs with decreased reflexes. Initial laboratory results showed severe hypokalemia (K $1.5 \mathrm{mEq} / \mathrm{L}$ ), Random blood sugar (RBS) of $400 \mathrm{mg} / \mathrm{dl}$, TSH: 5.68, Serum Renin $0.51 \mathrm{ng} / \mathrm{ml}$ (supine 0.15-2.33 ng/ml/hr, standing0.31$3.95 \mathrm{ng} / \mathrm{ml} / \mathrm{hr}$ ), Aldosterone $15 \mathrm{ng} / \mathrm{dl}$ (standing.4-31 ng/dl recumbent 1-16 ng/dl). His ACTH was $255 \mathrm{pg} / \mathrm{ml}, 24$ hour urine cortisol 9088 ug/24 hours. Serum cortisol was $192 \mathrm{ug} / \mathrm{dl}$, which failed to suppress with high dose $(8 \mathrm{mg})$ dexamethasone, remaining at $160 \mathrm{ug} / \mathrm{dl}$.

Findings on clinical examination and laboratory evaluation raised the suspicion of ectopic ACTH secretion originating from lungs. Chest imaging showed segmental consolidation/collapse of right anterobasal segment and bilateral pleural effusion (Figures 3 and 4). Subsequent bronchoscopy identified no lung mass but bronchoalveolar c/s grew Nocardia spp. (Table 2). MRI pituitary and $\mathrm{CT}$ abdomen showed no abnormality. Inferior petrosal sinus sampling showed no central-peripheral gradient (Table 3).

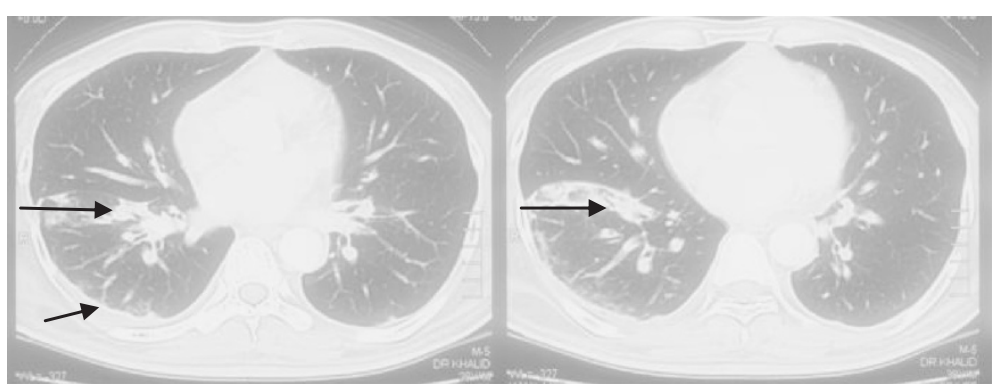

Figure 3 (Case 3) CT Chest before treatment for Nocardia (Consolidation right anterobasal segment \& pleural effusion). 


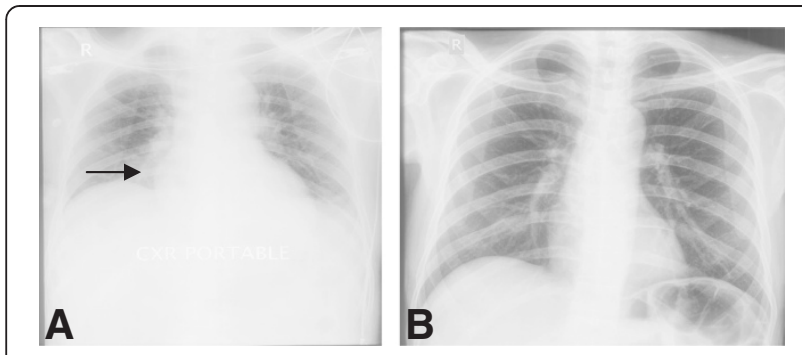

Figure 4 Chest X-ray before and after treatment for Nocardia (Case 3). (A) Pre-treatment consolidation right anterobasal segment \& pleural effusion. (B) Post-treatment resolution of right anterobasal segment consolidation and pleural effusion.

He was started on TS at $15 \mathrm{mg}$ trimethoprim/kg/day$930 \mathrm{mg} /$ day. Ketoconazole, potassium replacement, insulin and antihypertensive medication (aldactone and angiotensin receptor blocker) were optimized. On this regimen, the patient's condition improved and he was discharged home. On follow up visits, the doses of his medication were tapered down as his symptoms and biochemical profile improved. Follow-up chest x-ray was normal (Figure 4b). [Follow up CT scan was not done due to financial limitations]. To date (from 2011 to 2014), he has been off ketoconazole. His serum potassium and 24hour urine cortisol have remained within the normal range. His blood sugars are well- controlled off insulin therapy.

\section{Microbiological methods}

Nocardia was identified using standard phenotypic methods [5]. On gram stain from specimen, (Sputum, tracheal aspirates, Bronchoalveolar lavage), Nocardia was suspected when thin beaded Gram positive branching rods were seen. A modified partial acid fast stain was then performed. If these branching rods were partial acid fast positive, a preliminary report was issued stating "Gram positive branching rods that are partial acid fast positive suggestive of infection with Nocardia spp. are seen". Recommended quality control strains were used for Gram staining and partial acid fast staining. Growth of Nocardia spp was identified on colony

Table 3 Inferior petrosal sinus sampling (Case $\mathbf{3}$ )

\begin{tabular}{ll}
\hline ACTH Levels & $\mathbf{3 / 1 1 / 2 0 1 0}$ \\
\hline RT INF Petrosal 1 & 374 \\
RT INF Petrosal 2 & 356 \\
LT INF Petrosal 1 & 376 \\
LT INF Petrosal 2 & 371 \\
SVC & 358 \\
IVC & 326 \\
RT Peripheral & 356 \\
\hline
\end{tabular}

morphology, musty odor, gram stain morphology, partial acid fastness, presence of aerial hyphae, decomposition of casein, tyrosine, xanthine, hypoxanthine, urease production and gelatin hydrolysis. Antimicrobial susceptibility testing was performed using Kirby Bauer disc diffusion method [6]. S. aureus ATCC 29213, P. aeruginosa ATCC 27853 and E.coli ATCC 35218 (for amoxicillin-clavulanic acid) were used for quality control of susceptibility testing.

\section{Conclusions}

Our case series illustrates the different ways in which Nocardiosis can present in the setting of Ectopic Cushings. Ectopic Cushings is a well recognized cause of Cushing's syndrome, usually characterized by remarkably elevated levels of ACTH, elevated 24hour urine cortisol levels and severe/resistant hypokalemia, which can manifest as proximal myopathy [1,2]. All 3 subjects had uncontrolled hypertension and high blood sugars. They were hypokalemic (K: 2.52, 2.9, $1.5 \mathrm{mmol} / \mathrm{l}$ ); 24hour urine cortisol was elevated at 2000, 27216 and 9088 (32-243 ug/24 hours); ACTH 68.5, 159, 255 [0-48 pg/ml], respectively. Their MRI pituitary showed no adenoma, and inferior petrosal sinus sampling had revealed no central peripheral gradient.

In the first case, Nocardia spp produced a rapidly expanding cavitatory lesion in the left lung, and later left lower lobe collapse. The lung lesions eventually resolved with institution of appropriate antibiotic-trimethoprimsulphamethoxazole (TS). Nocardia failed to grow on culture. This could have been a consequence of previous antibiotic use. In the second case, left upper and lower lung cavitatory lesions were identified, with bronchial washings revealing growth of nocardia spp. Although appropriate coverage for Nocardia spp. with TS was given, followed by resolution of the lung lesions, the patient had underlying metastatic adenocarcinoma of unknown primary. This ultimately led to a rapid deterioration of his condition, and death. In the third case, CT chest revealed consolidation of right lung lobe and bilateral pleural effusion. Subsequent bronchoalveolar c/s grew Nocardia spp. In this patient, the patient's physical condition and biochemical profile, including the potassium level, 24 hour urine cortisol \& blood sugar, as well as pulmonary lesions, on follow-up chest X-ray, had completely normalized with the course of TS for nocardia spp. We were able to taper off his ketoconazole, potassium supplementation and insulin.

The remarkably elevated levels of ACTH usually encountered in ectopic Cushing's lead to high levels of glucocorticoid secretion. Glucocorticoids cause immunosupression by inhibiting the phagocytic function of alveolar macrophages and neutrophils, thereby reducing mobilization of inflammatory cells into infected areas. This results in an increased incidence of opportunistic 
bacterial and fungal infections [7]. Our case series highlights the importance of considering Nocardia spp as a causative agent for the pulmonic manifestations of patients diagnosed with Cushing's syndrome, particularly in the Ectopic Cushing's subset.

Opportunistic infections in Cushings syndrome carry a high mortality and morbidity $[2,8]$. A variety of opportunistic infections have been demonstrated in the presence of endogenous cortisol production. Most popular are Pneumocystis jirovecci, Cryptococcus neoformans and Nocardia spp. [9]. Huang TP et al. report a case of a young man who developed fever and dyspnea, with progression of his lung lesions, during the course of his hospital stay for work-up of Cushing's syndrome. Infection with Nocardia spp was found. Despite institution of appropriate antimicrobial therapy, the patient expired [8]. Graham BS et al. studied a series of patients with endogenous Cushings syndrome and opportunistic infections. They indicated that hypercortisolism may lead to masking of symptoms and signs of infection, at times leading to a delay in the diagnosis, which contributes to the high mortality rate [10]. Sutton BJ et al. report a case of Cushing's syndrome and nocardiosis associated with a pulmonary carcinoid tumour, diagnosed on fine needle aspiration of a left lung nodule. Prior to treatment for the tumour, new nodules developed in the lungs, fine needle aspiration of which revealed the nodules to be infectious in nature. Gram staining demonstrated gram positive branching organisms, and specimen culture grew Nocardia spp. [3]. Their case was amongst the first reporting use of the fine needle aspiration technique to diagnose both the pulmonary tumour, as well as the associated infectious complication arising from the hypercortisolism resulting from the tumour [3]. Boscaro $\mathrm{M}$ et al. report a patient with a disseminated form of nocardiosis in the setting of hypercortisolism resulting from bilateral nodular hyperplasia [11]. In a published series, ectopic production of ACTH has been responsible for the majority of opportunistic infections related to Cushing syndrome. This is most likely because the highest levels of glucocorticoid secretion are found in patients with ectopically produced ACTH $[8,9]$. Hoshino $\mathrm{T}$ et al. report a case of a young woman who presented with dyspnea. Chest X-ray examination showed an infiltrative shadow with pleural effusion. Pleural fluid obtained by thoracentesis showed Nocardia spp. Subsequently, she was diagnosed as a case of Cushings syndrome on the basis of low dose dexamethasone suppression test [12]. Sudou A et al. also report a case of pulmonary Nocardia otitidis-caviarum infection in a 35-year-old man with Cushing's disease. Chest radiograph showed a large bilateral mass in the lung fields. Sputum culture revealed Nocardia otitidis-caviarum [13]. Despite having received appropriate antibiotics, the patient expired due to respiratory failure. Tanaka $\mathrm{M}$ et al. also report a case of nocardia causing cerebral abscess in a patient with Cushing's syndrome. The patient was admitted with complaints of general fatigue, thirst and backache. A diagnosis of Cushing's syndrome was made on the basis of elevated serum levels of cortisol and adrenocorticotropic hormone (ACTH). Pituitary imaging was completely normal. During hospital stay, he developed hemiplegia. Cerebral abscess in the right frontal lobe was demonstrated by brain CT scan. Surgical drainage was performed and Nocardia spp was isolated from the drained pus [14].

Other opportunistic infections such as cryptococcosis, aspergillosis, pneumocystosis and risk of pulmonary involvement in the setting of herpes viral infections, such as Cytomegalovirus and Varicella zoster virus, also need to be considered in a patient with Ectopic Cushing's [8].

To the best of our knowledge, no case to date has been reported in the literature in which treatment of the Nocardia spp. resulted in disappearance of the clinical, biochemical and radiologic manifestations of the Ectopic Cushing's (as in case 3). This included the severe proximal myopathy, refractory hypokalemia, hypercortisolism and the pulmonary lesions that the patient had presented with. We were able to withdraw all therapy (including ketoconazole). The patient has remained symptom-free till date (years 2011 to 2014). This suggests that nocardiosis may not only result from hypercortisolism, but can also itself lead to raised cortisol levels, and the manifestations of Cushing's syndrome. The mechanism by which Nocardia spp. can cause Cushing's syndrome is not clear, and, therefore, needs to be investigated further.

Opportunistic infections are known to be associated with hypercortisolism, and higher levels of glucocorticoid secretion are found in patients with ectopically produced ACTH. Pulmonary nocardiosis is an important differential to consider. This series includes the first case reported in which the signs and symptoms of Cushing's subsided simply after antibiotic treatment of Nocardia.

\section{Consent}

Written informed consent was obtained from all three patients for publication of this case report. Copies of the written consent statements are available for review by the Series Editor of this journal.

\section{Abbreviations}

ACTH: Adrenocorticotrophic hormone; TS: Trimethoprim-sSulphamethoxole; IPSS: inferior petrosal sinus sampling; AFBC/S: Acid fast bacillus culture \& sensitivity; FBS: Fasting blood sugar; RBS: Random blood sugar; TSH: Thyroid stimulating hormone; Nocardia spp: Nocardia species; CPR: Cardiopulmonary resuscitation.

\section{Competing interests}

The authors declare that they have no competing interests. 


\section{Authors' contributions}

$A R$ and $A S$ led the conception and design, acquisition of data, review of literature, and drafted the manuscript. NI, AJ and JA reviewed the manuscript and gave the concept of research paper, and critically reviewed the manuscript. All authors read and approved the manuscript.

\section{Authors' information}

AR is Fellow of the College of Physicians \& Surgeons of Pakistan. She is Consultant in Endocrinology, Diabetes \& Metabolism, Department of Medicine and Masters in Clinical Research, Aga Khan University Hospital. She was involved in the medical management of the first two patients. AS is Fellow of the College of Physicians \& Surgeons of Pakistan. She is Fellow in Endocrinology, Diabetes \& Metabolism, Department of Medicine, Aga Khan University Hospital. She was involved in the medical management of the third patient. AJ is Fellow of the Royal College of Physicians of London. He is Professor \& Consultant Endocrinologist, Department of Medicine, Aga Khan University Hospital. He was the second patient's primary physician \& endocrinologist. JA is Fellow of the Royal College of Physicians of London. $\mathrm{He}$ is Professor \& Consultant Endocrinologist, Department of Medicine, Aga Khan University Hospital. He was the third patient's primary physician \& endocrinologist. NI is Fellow of the Royal College of Physicians of London. He is Professor \& Consultant Endocrinologist, Department of Medicine, Aga Khan University Hospital. He is also the Director Endocrinology, Diabetes and Metabolism Fellowship Program, Aga Khan University Hospital. He was the first patient's primary physician \& endocrinologist.

\section{Acknowledgements}

We thank Kauser Jabeen (Assistant Professor, Microbiology, AKUH) on providing immense assistance with details on microbiological data. We thank Basit Salam and Zainab Hussain (consultant radiologists, AKUH) for providing assistance on appropriate CT scan slide selection.

Received: 30 October 2013 Accepted: 2 June 2014

Published: 20 June 2014

\section{References}

1. Nieman LK, Biller BM, Findling JW, Price JN, Savage MO, Stewart PM, Montori VM: The diagnosis of Cushing's syndrome: an Endocrine Society Clinical Practice Guideline. J Clin Endocrinol Metab 2008, 93(5):1526-1540.

2. Ilias I, Torpy DJ, Pacak K, Mullen N, Wesley RA, Nieman LK: Cushing's Syndrome Due to Ectopic Corticotropin Secretion: Twenty Years' Experience at the National Institutes of Health. J Clin Endocrinol Metab 2005, 90(8):4955-4962.

3. Sutton BJ, Parks GE, Manavi CK, Palavecino EL, Geisinger KR: Cushing's syndrome and Nocardiosis Associated with a Pulmonary Carcinoid Tumour: Report of a Case and Review of the literature. Diagn Cytopathol 2011, 39(5):359-362.

4. Harris K, Khachaturova I, Azab B, Maniatis T, Murukutla S, Chalhoub M, Hatoum H, Kilkenny T, Elsayegh D, Maroun R, Alkaied H: Small Cell Lung Cancer Doubling Time and its Effect on Clinical Presentation: A Concise Review. Clin Med Insights Oncol 2012, 6:199-203.

5. Koneman EW, Allen SD, Janda WM, Schreckenberger PC, Winn WC: Color atlas and textbook of diagnostic microbiology. 6th edition. Philadelphia, PA: Lippincott; 2005.

6. Kiska DL, Hicks K, Pettit DJ: Identification of medically relevant Nocardia species with an abbreviated battery of tests. J Clin Microbiol 2002, 40(4):1346-1351.

7. Fauci AS, Dale DC, Balow JE: Glucocorticosteroid therapy: mechanisms of action and clinical considerations. Ann Intern Med 1976, 84(3):304-315.

8. Bakker RC, Gallas PR, Romijn JA, Wiersinga WM: Cushing's syndrome complicated by multiple opportunistic infections. J Endocrinol Invest 1998, 21(5):329-333.

9. Beinart GA, Rao RK, Hollander H: Ectopic ACTH Syndrome Resulting in Nocardiosis and Acute Respiratory Failure. Hosp Physician 2003, 39(10):49-54.

10. Graham BS, Tucker WS: Opportunistic infections in endogenous Cushing's syndrome. Ann Intern Med 1984, 101(3):334-338.

11. Boscaro M, Fallo F, Sonino N: Disseminated nocardiosis in a patient with Cushing's syndrome. J Endocrinol Invest 1994, 17(6):443-445.

12. Hoshino T, Okamoto M, Azuma K, Toda R, Kawayama T, Kato T, Yamada K, Aizawa $\mathrm{H}$ : A case of Cushing syndrome presenting after pulmonary nocardiosis with pyothorax. Nihon Kokyuki Gakkai Zasshi 2009, 47(8):746-750

13. Sudou A, Hashimoto T, Nakamura H, Yagyuu H, Sarashina G, Hatao E, Tuchida F, Adachi H, Kishi K, Matuoka T: Pulmonary Nocardia otitidis-caviarum infection in a patient with Cushing's disease. Nihon Kokyuki Gakkai Zasshi 2001, 39(3):210-214.

14. Tanaka M, Sato Y, Ito H, Ichikawa Y, Oizumi K, Shigemori M: A case of Cushing's syndrome associated with Nocardia cerebral abscess. Kansenshogaku Zasshi 1991, 65(2):243-249.

doi:10.1186/1472-6823-14-51

Cite this article as: Rizwan et al:: Case report: nocardia infection

associated with ectopic cushings. BMC Endocrine Disorders 2014 14:51.

\section{Submit your next manuscript to BioMed Central and take full advantage of:}

- Convenient online submission

- Thorough peer review

- No space constraints or color figure charges

- Immediate publication on acceptance

- Inclusion in PubMed, CAS, Scopus and Google Scholar

- Research which is freely available for redistribution

Submit your manuscript at www.biomedcentral.com/submit
C BioMed Central 